\title{
Algoritmo para el manejo de pacientes con uropatía obstructiva secundaria a cáncer de próstata
}

\author{
Urdaneta Pignalosa G, Rodríguez Faba O, Palou Redorta J, Rosales Bordes A, \\ Esquena Fernández S, Villavicencio Mavrich H.
}

Servicio de Urología. Fundació Puigvert. Barcelona.

Actas Urol Esp. 2008;32(2):179-183

\section{RESUMEN \\ ALGORITMO PARA EL MANEJO DE PACIENTES CON UROPATÍA OBSTRUCTIVA SECUNDARIA \\ A CÁNCER DE PRÓSTATA}

Introducción: Una de las complicaciones de la progresión del cáncer de próstata es la uropatía obstructiva, por infiltración y compresión del tramo ureteral distal, que puede conllevar a una insuficiencia renal aguda, con afectación de la calidad de vida y la supervivencia de estos pacientes.

El tratamiento del cáncer de próstata con ureterohidronefrosis secundaria es paliativo y siguiendo las tendencias actuales, se debe considerar la colocación de una nefrostomía.

Materiales y Métodos: Se realizó una búsqueda en PUBMED y se revisaron los artículos más representativos. El algoritmo se construyó con base en la práctica clínica diaria basada en la rutina, el protocolo de nuestro centro y con la evidencia científica disponible en la literatura médica.

Resultados: Se propone un algoritmo de decisiones para definir la derivación urinaria en pacientes con uropatía obstructiva secundaria a cáncer de próstata.

Conclusiones: La indicación de colocar una nefrostomía en pacientes con uropatía obstructiva secundaria a cáncer de próstata debe abordarse individualmente, de acuerdo a las condiciones generales y la calidad de vida del paciente con base en escalas ya definidas en la literatura (ECOG y Karnofsky) y en factores de buen o mal pronóstico, siempre teniendo en cuenta consideraciones éticas y el consentimiento del paciente y de su familia.

Palabras clave: Obstrucción ureteral. Nefrostomía. Neoplasias prostáticas/complicaciones. Uropatía obstructiva.

\section{ABSTRACT \\ ALGORITHM FOR THE TREATMENT OF PATIENTS WITH OBSTRUCTIVE UROPATHY SECONDARY TO PROSTATE CANCER}

Background: One of the prostate cancer progression complications is the obstructive uropathy, by infiltration and compression of the distal ureteral section, that can entail to an acute renal insufficiency, with affectation of the quality of life and the survival of these patients.

The treatment of prostate cancer with secondary ureterohidronefrosis is palliative and following the present tendencies, the positioning of a nephrostomy is considered.

Materials and Methods: A search was made in PUBMED and the most representative articles were reviewed. The algorithm was constructed with the daily routine clinical base, the protocol of our center and with the scientific evidence available in medical literature.

Results: An algorithm of decisions sets out to define the urinary derivation in patients with obstructive uropathy secondary to prostate cancer.

Conclusions: The indication to place a nephrostomy in patients with obstructive uropathy secondary to prostate cancer must be approached individually, according to the general conditions and the quality of life of the patient with base in scales defined in literature (ECOG and Karnofsky) and in factors of good or bad prognosis, always considering ethical considerations and the consent of the patient and his family.

Keywords: Ureteral obstruction. Nephrostomy. Prostatic neoplasms/complications. Obstructive uropathy. 
$\mathrm{E}$ n España, durante los últimos años, el cáncer de próstata se ha colocado en el tercer lugar como causa de muerte en hombres por detrás de los tumores de pulmón y de los colorrectales $^{1}$. Su comportamiento clínico, respuesta terapéutica y supervivencia son heterogéneos y dependen de muchos factores.

La progresión del cáncer de próstata se puede manifestar con exacerbación de síntomas locales, diseminación metastásica principalmente ósea y complicaciones diversas como la uropatía obstructiva ( 3,3 y $16 \%$ ), por infiltración y compresión del tramo distal de los uréteres, generando insuficiencia renal aguda; lo que tiene un impacto considerable en la calidad de vida y la supervivencia de estos pacientes ${ }^{2}$.

Históricamente, el pronóstico para pacientes en esta situación oncológica es pobre y su supervivencia media dependerá de si están en fase hormonorefractaria (media 7 meses) o son pacientes hormonalmente naif (media 24 meses) ${ }^{3}$.

Un estadio avanzado y/o una enfermedad hormonorefractaria se correlacionan significativamente con el desarrollo de uropatía obstructiva que está asociada con una reducción significativa de la supervivencia global comparada con los pacientes con cáncer de próstata sin obstrucción.

El tratamiento del cáncer de próstata con ureterohidronefrosis secundaria suele ser paliativo y las tendencias actuales abogan por la colocación de nefrostomía a todos los pacientes hormonalmente naif con uropatía obstructiva, mientras que los hormonoresistentes requieren un abordaje individualizado debido al escaso aumento de la supervivencia (115 dias) ${ }^{4}$.

\section{MATERIALES Y MÉTODOS}

Se realizó una búsqueda en PUBMED (www.pubmed.com) con los términos "obstructive uropathy" AND "nephrostomy" y se revisaron los artículos más representativos. Igualmente se consultaron libros-guía de urología.

El algoritmo (Fig. 1) se construyó con base en la práctica clínica diaria basada en la rutina y protocolo de nuestro centro y la evidencia científica disponible en la literatura médica revisada.

El objetivo es generar una guía de decisiones lo más consensuadas posibles para atender un razonamiento estructurado y ser un instrumento

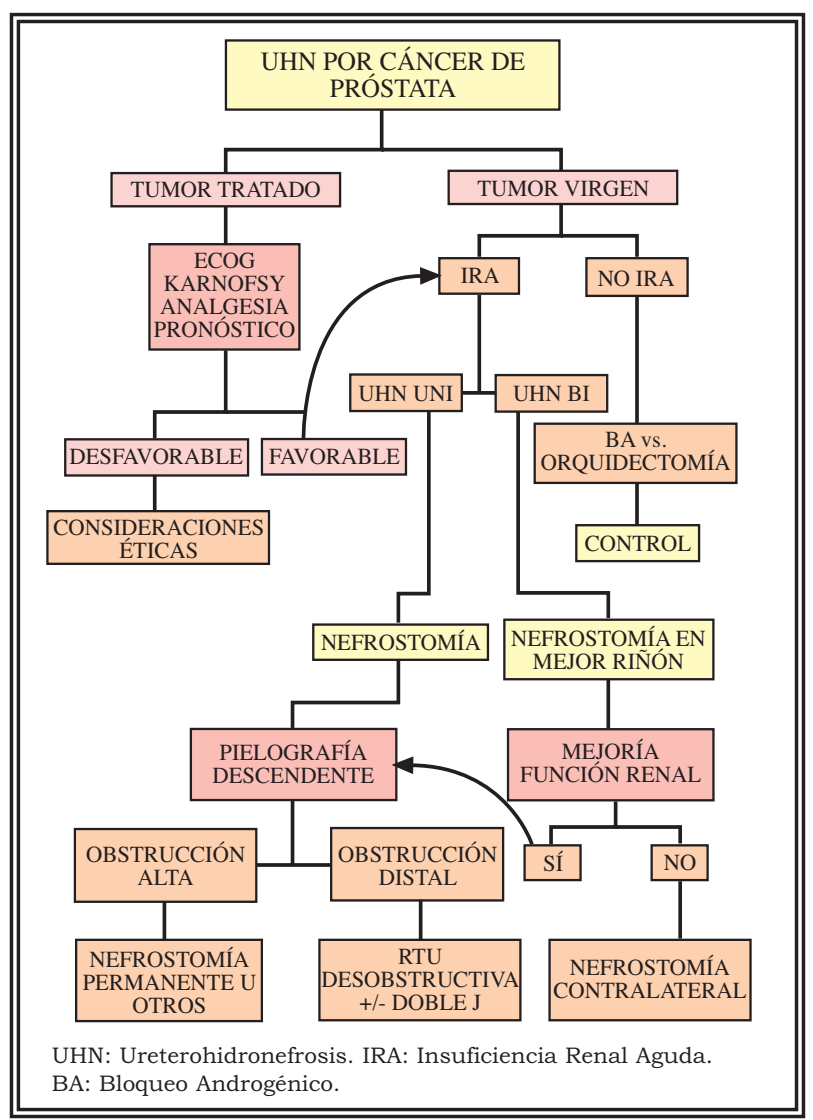

FIGURA 1. Algoritmo para el manejo de pacientes con uropatía obstructiva secundaria a cáncer de próstata.

de ayuda en el proceso de toma de decisiones. Se propone una guía clínica incorporando una secuencia sistematizada de actividades entre las variaciones inapropiadas de la práctica clínica, facilitando la buena praxis.

\section{DISCUSIÓN}

Según la literatura, la mayoría de las obstrucciones ureterales por causa neoplásica ocurren por infiltración o compresión del tramo distal ureteral (neoplasia vesical, prostática o de cérvix).

La obstrucción al flujo de la orina de uno o ambos uréteres también puede deberse a compresión extrínseca por una neoplasia o adenopatías (linfomas, metástasis ganglionares). Otra causa posible es la fibrosis que se origina tras cirugía o radioterapia en el retroperitoneo

En cuanto a la asociación de uropatía obstructiva y cáncer de próstata, ésta se da en estadios localmente avanzados o metastáticos y ensombrece el pronóstico del paciente. Sin embargo, la obstrucción ureteral asociada con el cáncer 
de próstata y de cuello uterino usualmente tienen un mejor pronóstico que otros tipos de neoplasia con un incremento en la supervivencia, de 1 año o más en aproximadamente $60 \%$ de los pacientes ${ }^{5,6}$; razón por la cual se considera fundamental la derivación urinaria alta como una opción a tener en cuenta en estos pacientes, individualizando la indicación en cada caso.

Como precepto hay que tener en cuenta que la derivación urinaria alta puede mejorar la calidad de vida evitando la insuficiencia renal aguda y por consecuencia el deterioro clínico dado por la uremia ${ }^{7}$.

Es imprescindible la realización de un buen diagnóstico para considerar la derivación urinaria. Principalmente, se trata de pacientes que consultan al servicio de urgencias por disminución de la diuresis, síntomas derivados de un fracaso renal agudo o que presentan deterioro progresivo de la función renal detectado en los sucesivos controles.

Finalmente el diagnóstico se confirma radiológicamente con ultrasonido principalmente o TAC, que documenta la ectasia de la vía urinaria alta y la ausencia de orina en vejiga.

Aunque es un procedimiento seguro, fácil técnicamente y con escasas complicaciones (mala colocación, sangrado e infecciones) puede estar contraindicado (Tabla 1$)^{8}$. La decisión debe ser considerada con el consentimiento del paciente y/o de su familia para realizarla o no, individualizando cada caso ${ }^{8}$.

Adicionalmente se debe tener claro si es un tumor virgen o tratado. En el primer caso se puede asumir que es un paciente en el cual la deprivación androgénica puede reducir el tamaño tumoral y la vascularización al igual que hacer más fácil la posterior RTU desobstructiva e inclusive mejorar el síndrome obstructivo urinario bajo ${ }^{9}$; por lo cual está indicado iniciar el Bloqueo Androgénico Farmacológico (BAF) o la propuesta de una orquidectomía simple bilateral en aquellos enfermos con mala adherencia al tratamiento farmacológico. Sin embargo, si existe IRA se les puede ofrecer una nefrostomía que puede ser retirada si se produce la repermeabilización ureteral.

En el caso de tumores tratados es importante valorar diferentes factores pronósticos para tomar la decisión. En primer lugar se debe valorar el estado físico del paciente (nivel de hemoglobina, PSA y LDH) y su nivel de actividad; para lo cual se han desarrollado escalas para determinar como la enfermedad afecta sus capacidades (ECOG: Eastern Cooperative Oncology Group ${ }^{10}$ y la Escala de Karnofsky ${ }^{11}$ ). En este sentido es importante valorar el grado de dolor que presenta el paciente en la evolución de su enfermedad metastásica y requerimiento analgésico diario.

Según Huguet et al. ${ }^{12}$ se ha observado un peor pronóstico de forma significativa en pacientes con ECOG $>2$, Karnofsky $<80 \%, \mathrm{Hb}<10-12 \mathrm{~g} / \mathrm{dL}$, PSA > $100 \mathrm{~g} / \mathrm{dL}$ y por esta razón es importante tenerlo en cuenta en el algoritmo al tomar la decisión de realizar una derivación urinaria.

Posteriormente se debe documentar si la obstrucción es unilateral o bilateral teniendo en cuenta que es bilateral en más del $50 \%$ de los pacientes ${ }^{13}$. De acuerdo a esto, tomar una conducta para saber cual es el riñón que se debe derivar en primera instancia. Definitivamente la recomendación es realizarla en el riñón que radiológicamente posea mejor función (mejor parénquima, más función) para luego tomar la decisión de además derivar el contralateral si así se requiere.

Tabla 1. Contraindicaciones para la derivación urinaria (modificada de Mohamed Mohamed-Abdallah $Z$ et al.).

\begin{tabular}{ll}
\hline Pacientes con dudosa indicación para la derivación: & $\begin{array}{l}\text { Enfermos que no deben ser derivados (salvo insistencia } \\
\text { del enfermo o su familia): }\end{array}$ \\
\hline $\begin{array}{l}\text { Enfemedad metastásica con posibilidad de supervivencia } \\
\text { de meses. }\end{array}$ & $\begin{array}{l}\text { Tumores recidivados y/o diseminados a pesar del } \\
\text { tratamiento oncológico completo (cirugia, radioterapia, } \\
\text { quimioterapia). }\end{array}$ \\
Historia de coagulopatías. & $\begin{array}{l}\text { Tumores de rápida progresión a pesar del tratamiento } \\
\text { adecuado. }\end{array}$ \\
Paciente en anticoagulación. & $\begin{array}{l}\text { Grave alteración del estado psico-emocional. } \\
\text { Requerimiento analgésico alto. }\end{array}$ \\
\hline
\end{tabular}


Finalmente, teniendo como objetivo principal la comodidad y el confort del paciente; se debe intentar, siempre y cuando sea posible, el cambio de la nefrostomía simple a una prótesis más anatómica, menos expuesta al exterior y más fácil de conllevar como lo es un catéter doble $\mathrm{J} \mathrm{y}$, con este fin, se recomienda la realización de una pielografía descendente o anterógrada para documentar el nivel de la obstrucción urinaria causante de la UHN y su grado de permeabilidad.

Incluso existen recientes trabajos sobre la utilización de nuevos stents metálicos de composición variable en pacientes con obstrucción ureteral extrínseca maligna, en situación final de su enfermedad y parece ser que en los recubiertos de materiales inertes como el Dacron, se incrementa su durabilidad, incluso en más de 9 meses. Además, presentan mucha mayor resistencia que los de silicona, evitando el colapso durante el tiempo de uso ${ }^{14-16}$

Sin embargo, al comparar los pacientes que llevan catéter doble $\mathrm{J}$ y los que llevan sonda de nefrostomía se demuestra mayor disconfort y clínica irritativa vesical en portadores de doble $\mathrm{J}$ y no se demuestran diferencias significativas en cuanto a la calidad de vida global (QoL) de estos pacientes ${ }^{17}$. Adicionalmente existen casos en que una derivación retrógrada tipo catéter doble $\mathrm{J}$ no se le puede ofrecer al paciente pues es técnicamente difícil o imposible (más del $50 \%)^{18}$, en la presencia de compromiso pélvico avanzado por procesos malignos, y se debe indicar como primera medida la nefrostomía a permanencia. Sin embargo, como lo recomiendan Banús et al. se puede realizar una RTU desobstructiva de los meatos ureterales comprometidos y colocar un catéter doble $\mathrm{J}$ retrógrado, temporal o definitivo, e inclusive realizar una RTU de próstata para evitar residuo de orina e infección secundaria ${ }^{19}$.

\section{CONCLUSIONES}

La uropatía obstructiva por cáncer de próstata avanzado varía entre 3,3 y $16 \%{ }^{2}$. Históricamente, el pronóstico para pacientes en esta situación oncológica es pobre.

La uropatía obstructiva secundaria al cáncer de próstata está asociada con una reducción significativa de la supervivencia global comparada con los pacientes con cáncer de próstata sin obstrucción.
Un estadio avanzado de la enfermedad se correlaciona significativamente con el desarrollo de uropatía obstructiva ${ }^{20}$. Según lo anterior y de acuerdo a la revisión, se puede recomendar que todos los pacientes deben ser candidatos a nefrostomía sin importar su estado hormonal ${ }^{3}$ y la nefrostomía debe colocarse, si está indicada, lo más tempranamente posible para evitar un incremento de la mortalidad por complicaciones urémicas.

Ante una posible abstención terapéutica se debe considerar si la enfermedad está en fase terminal sin posibilidad de tratamiento curativo, con un requerimiento analgésico elevado y mal estado general (Índice de Karnofsky).

\section{REFERENCIAS}

1. Centro Nacional de Epidemiología. Instituto de Salud Carlos III. Área de Epidemiología Ambiental y Cáncer. La situación del cáncer en España.

2. James ND, Bloomfield D, Luscombe C. The changing pattern of management for hormone-refractory, metastatic prostate cancer. Prostate Cancer Prostatic Dis. 2006;9(3): 221-229.

3. Colombel M, Mallame W, Abbou CC. Influence of urological complications on the prognosis of prostate cancer. Eur Urol. 1997;31 Suppl 3:21-24.

4. Harris MR, Speakman MJ. Nephrostomies in obstructive uropathy; how should hormone resistant prostate cancer patients be managed and can we predict who will benefit? Prostate Cancer Prostatic Dis. 2006;9(1):42-44.

5. Chiou RK, Chang WY, Horan JJ. Ureteral obstruction associated with prostate cancer: the outcome after percutaneous nephrostomy. J Urol. 1990;143(5):957-959.

6. Wilson JR, Urwin GH, Stower MJ. The role of percutaneous nephrostomy in malignant ureteric obstruction. Ann R Coll Surg Engl. 2005;87(1):21-24.

7. Logothetis CJ, Hey DM. Urologic Complications. In Holland-Frei CANCER MEDICINE 7. Kufe DW, Bast RC, Hait WN, Hong WK, Pollock RE, Weichselbaum RR. Philadelphia. B.C. Decker, Chapter 133. 2006.

8. Mohamed-Abdallah ZM, Corral J, Fuertes ME, et al: Derivaciones urinarias del tracto urinario superior en el anciano. Clínicas Urológicas de la Complutense 1993;2:353-60.

9. Crain DS, Amling CL, Kane CJ. Palliative transurethral prostate resection for bladder outlet obstruction in patients with locally advanced prostate cancer. J Urol. 2004;171(2 Pt 1):668-671.

10. Oken MM, Creech RH, Tormey DC, HortonJ, Davis TE, McFadden ET, Carbone PP. Toxicity and response criteria of the Eastern Cooperative Oncology Group. Am J Clin Oncol. 1982;5(6):649-655.

11. Karnofsky DA, Abelmann WH, Craver LF, Burchenal JH. The use of the nitrogen mustards in the palliative treatment of carcinoma. With particular reference to bronchogenic carcinoma. Cancer. 1948;1(4):634-656.

12. Huguet Pérez J, Maroto Rey P, Palou Redorta J, Villavicencio Mavrich H. Cáncer de próstata hormonorresistente. Cambios en las estrategias terapéuticas desde la demostración de la utilidad de la quimioterapia. Actas Urol Esp. 2006;30(2):123-133. 
13. Smith JA Jr, Soloway MS, Young MJ. Complications of advanced prostate cancer. Urology. 1999;54(6A Suppl):814.

14. Borin JF, Melamud O, Clayman RV. Initial experience with full-length metal stent to relieve malignant ureteral obstruction. J Endourol. 2006;20(5):300-304.

15. Pandian SS, Hussey JK, McClinton S. Metallic ureteric stents: early experience. Br J Urol. 1998;82(6):791-797.

16. Tekin MI, Aytekin C, Aygün C, PeSkircio€lu L, Boyvat F, Ozkardefl $\mathrm{H}$. Covered metallic ureteral stent in the management of malignant ureteral obstruction: preliminary results. Urology. 2001;58(6):919-923.

17. Joshi HB, Adams S, Obadeyi OO, Rao PN. Nephrostomy tube or 'JJ' ureteric stent in ureteric obstruction: assessment of patient perspectives using quality-of-life survey and utility analysis. Eur Urol. 2001;39(6):695-701.
18. Uthappa MC, Cowan NC. Retrograde or antegrade doublepigtail stent placement for malignant ureteric obstruction?. Clin Radiol. 2005;60(5):608-612.

19. Banús Gassol JM, Palou Redorta J, Cortadellas Angel L, Morote Robles J, Soler Roselló A. Transurethral Resection of the Ureteral Meatus Invaded by Carcinoma of the Prostate: A New Approach. Eur Urol. 1987;13(5):344-345.

20. Oefelein MG. Prognostic significance of obstructive uropathy in advanced prostate cancer. Urology. 2004;63(6):1117-1121.

Correspondencia autor: Dr. G. Urdaneta Pignalosa Servicio de Urología. Fundació Puigvert

Cartagena, 340-350 - 08025 Barcelona. Tel.: 934169700

E-mail autor: gurdaneta@fundacio-puigvert.es

Información artículo: Original - Cáncer de próstata

Trabajo recibido: enero 2007

Trabajo aceptado: junio 2007 